\title{
Measurements of C-Reactive Protein for Successful Management and Follow-Up Treatment of Neonatal Sepsis and Nosocomial Infection
}

\author{
Yasser Z. El-Nahhal*, Ahmed T. Al_Shareef, Mohammed R. Alagha \\ Department of Toxicology, Faculty of Science, The Islamic University of Gaza, Gaza Strip, Palestine \\ Email: *y_el_nahhal@hotmail.com
}

How to cite this paper: El-Nahhal, Y.Z., Al_Shareef, A.T. and Alagha, M.R. (2019) Measurements of C-Reactive Protein for Successful Management and Follow-Up Treatment of Neonatal Sepsis and Nosocomial Infection. Health, 11, 721-732. https://doi.org/10.4236/health.2019.116060

Received: April 22, 2019

Accepted: June 8, 2019

Published: June 11, 2019

Copyright $\odot 2019$ by author(s) and Scientific Research Publishing Inc. This work is licensed under the Creative Commons Attribution International License (CC BY 4.0).

http://creativecommons.org/licenses/by/4.0/

\begin{abstract}
Objective: To develop an optimal follow-up treatment for neonatal and nosocomial infections and to examine correlations between the C-reactive protein (C-RP) and platelets. Methods. A sample of 27 septic neonates and 14 non-septic neonates were selected for this study. The non-septic neonates served as a control group. Blood samples were collected from both groups and analyzed for bacterial contamination via blood culture, complete blood count (CBC), and C-RP, according to international laboratory standards. Blood collection and analysis were repeated every day during the follow-up treatments with antibiotics to evaluate the kinetics of C-RP. Results: Blood culture found E. coli and Staphylococcus aureus in the blood of the septic cases. Serum C-RP concentrations were at high levels $(24 \mathrm{mg} / \mathrm{dl})$ in the septic neonates and at normal levels (lower than $6 \mathrm{mg} / \mathrm{dl}$ ) in the control group. Treatment with active antibiotics resulted in a drastic reduction of the C-RP values and helped to reach a normal level as in the control group. On the other hand, there were increases in the platelet levels as the C-RP levels decreased. This result indicates a strong negative association between C-RP and platelet levels in the septic group only. Statistical analysis shows significant differences between the mean C-RP serum concentrations of the sepsis and non-sepsis. Conclusion: blood culture, active antibiotics, and kinetic C-RP measurements during the medical follow-up treatment are strong driving parameters for the optimal and successful management of sepsis.
\end{abstract}

\section{Keywords}

Blood Culture, Active Antibiotics, C-RP, Neonatal Sepsis 


\section{Introduction}

Sepsis is a major cause of death in intensive care units [1]. Its early diagnosis and timely antimicrobial treatment are driving parameters for the improvement of nosocomial and neonatal septic cases [2]. Early diagnosis of sepsis may be a challenging element since inflammation may be caused by pathogenic microbes [3] and/or environmental factors [4] [5]. Currently, C-reactive protein (C-RP) is a widely used marker of inflammation. It is synthesized by liver cells in response to pro-inflammatory cytokines [6]. Concentrations of C-RP in blood are very low in healthy individuals but may rapidly be increased after induction of an inflammatory response associated with bacterial infections. This property makes $\mathrm{C}-\mathrm{RP}$ an important marker to detect many inflammatory diseases. For instance, it has been used to predict human lung epithelial cells [7], impaired lung function [8], oxidative stress [9], bronchiolitis obliterans syndrome [10], pulmonary diseases [11], bronchial hyperresponsiveness [12], chronic obstructive pulmonary disease [13], lung cancer [14], asthma, [15] and neonatal sepsis [16].

Successful management of sepsis among neonates depends on the experience of the physicians, in many cases visual rating of patient features play a critical role in the medical treatment. In case of limited experience, physicians change the used antibiotics with different types to get successful management. This step may create antibiotic-microbial resistant species, or reduce patient immunity. Nevertheless, quantitate measures evaluating the optimal management of sepsis among neonates is not available. Furthermore, the abovementioned studies are limited for determining C-RP concentrations in the relationship with inflammation only without any follow-up and kinetic determinations during the medical treatment. Furthermore, a correlation between C-RP levels and CBC contents such as platelets and/or white blood cells (WBC) remains poorly investigated. The author of this study emphasized the importance of dynamic measurements of C-RP during medical treatment and follow-up of neonatal and nosocomial sepsis to some cases from the intensive baby care unit in the hospital of south Gaza and provided quantitative measures for the optimal managements.

\section{Materials and Methods}

\subsection{Sample Size}

The population of the sample size was calculated using Google sample size calculator (http://www.raosoft.com/samplesize.html 2018) in which the sample size are 41 septic neonates. All cases were recorded during seven months of the study period. The control group involved 14 sick neonates with physiological symptoms, such as jaundice who visited the hospital during the medical treatment of the groups. The septic cases were 14 nosocomial and 13 neonatal sepsis, in total 27 cases. Furthermore, the parents of the sepsis and non-sepsis cases were interviewed for data collection.

Cases of neonatal sepsis were characterized by signs and symptoms of infec- 
tion with or without accompanying bacteremia in the first 72 hours of age. It encompasses various systemic infections of the newborn such as septicemia, meningitis, pneumonia, arthritis, osteomyelitis, and urinary tract infections.

On the other hands, case of nosocomial (hospital-acquired) or community-acquired and neonates usually present with septicemia, pneumonia or meningitis after 72 hours of age up to 28 days.

\subsection{Observing Clinical Symptoms}

The clinical symptoms and signs of the cases were observed in accordance with international standards for septic cases [17]. The observed symptoms included baby skin color, baby activity, vomiting, urine, stool and feeding methods and amounts. These symptoms were recorded during 24 hours according to the internal method of the WHO [18].

\subsection{Blood Collections and Analysis}

Five $\mathrm{ml}$ blood sample was collected every day from each case during the medical treatment which lasted in 8 days. The collected blood was used for complete blood count (CBC), C-RP, blood culture, and liver function test, in accordance with international laboratory measurements [19] using commercially available kits.

\subsection{Antibiotic Microbial Response Tests}

Dose response relationships were conducted in the hospital laboratory in accordance with the international method [20].

\subsection{Management and Medical Follow-Up of the Cases}

Three well qualified medical teams from the main hospital in Gaza were taking care of the medical requirements and follow up of the cases according to the international methods during $24 \mathrm{~h}$.

Ethical issues: The study was approved by a local Ethical committee at ministry of health. Compliance with Ethical Standards was full filed and conflict of interest form was filled.

\subsection{Statistical Analysis}

The study consisted of three groups. Nosocomial, neonatal and control group. Blood parameters such as $\mathrm{HB}, \mathrm{WBC}$, Platelets, and C-RP were measured every day. Means and standard deviation of blood parameters in each group were calculated using Excel program. The statistical analysis was conducted by the t-test to detect differences between C-RP and platelets of the sepsis and control groups. A p-value $\leq 0.05$ indicates significant differences where further medical treatments and follow-up are necessary for the septic cases. In case of p-value > 0.05 , no further medical treatment or follow-up is required, and patients can be released from the hospital. 
The study was approved by the research committee of the Toxicology department at the Faculty of Science, the Islamic University-Gaza and by Helsinki Committee at the Ministry of Health. Consent form was obtained from the parents of the neonates.

\section{Results}

\subsection{Physical Feature of the Cases}

The population size of the cases was 27 in which 14 of them are nosocomial infection cases and 13 neonatal sepsis cases. The control group was 14 non-septic neonates. The physical features of these cases are shown in Table 1. It is obvious that patients affected by nosocomial infection cases, neonate sepsis and the control group have different body weight, temperature, skin color, activity and different feeding modes. The gender distribution is not significantly different between the three groups.

Table 1. Physical features and clinical symptoms and signs. Values in brackets indicate \% of the case under investigation.

\begin{tabular}{|c|c|c|c|}
\hline Baby item & $\begin{array}{c}\text { Nosocomial } \\
\text { infection }(n=14)\end{array}$ & $\begin{array}{l}\text { Neonatal sepsis } \\
\quad(\mathrm{n}=13)\end{array}$ & $\begin{array}{c}\text { Control group } \\
(\mathrm{n}=14)\end{array}$ \\
\hline $\begin{array}{l}\text { Weight } \\
\text { range }(\mathrm{kg})\end{array}$ & $1.75-2.8$ & $2.9-3.4$ & $2.9-3.5$ \\
\hline $\begin{array}{l}\text { Temperature } \\
\text { range }\left({ }^{\circ} \mathrm{C}\right)\end{array}$ & $36-39$ & $36-39$ & $36.5-37.5$ \\
\hline $\begin{array}{l}\text { Gestational } \\
\text { age (week) }\end{array}$ & $32-35$ & $33-36$ & 38 \\
\hline Skin color & pink & cyanotic & normal \\
\hline Male & $8(19.5)$ & $7(17.1)$ & $9(22)$ \\
\hline Female & $6(14.6)$ & $6(14.6)$ & $5(12.2)$ \\
\hline Baby activity & active & hypoactive & active \\
\hline Nasogastric tube & + & + & - \\
\hline Lung collapse & $9(22)$ & $5(12.2)$ & 0 \\
\hline Cardiomegaly & $3(7.3)$ & $1(2.44)$ & 0 \\
\hline Hepatomegaly & $6(14.6)$ & $6(14.6)$ & 0 \\
\hline Heart beat & $65-146$ & $48-65$ & $60-82$ \\
\hline Jaundice & $8(19.5)$ & $10(24.4)$ & $13(31.7)$ \\
\hline Blood culture & + & + & - \\
\hline $\begin{array}{c}\text { Staphylococcus } \\
\text { aureus }\end{array}$ & 00 & $13(31.7)$ & 0 \\
\hline E. coli & $6(14.63)$ & 00 & 0 \\
\hline Klebsiella spp. & $8(19.5)$ & 00 & \\
\hline
\end{tabular}




\subsection{Observed Clinical Symptoms}

The observed clinical symptoms included a collapsed lung in which represents 22 and $12.2 \%$ in the nosocomial infections and neonate sepsis cases, respectively. On the other hand, the percentage of cardiomegaly was higher in the nosocomial infections than in neonate sepsis cases. Furthermore, the percentage of hepatomegaly is nearly equal in nosocomial infections and neonate sepsis cases. Additionally, the central nervous system does not show any abnormality in all cases. The heartbeats are in different ranges in the septic group and control group. The percentages of jaundice were $19.5 \%, 24.4 \%$ and $31.7 \%$ in nosocomial infections, neonate sepsis cases and the control group, respectively.

\subsection{Kinetic Measurement of Blood Parameters during the Medical Treatment}

Hemoglobin (HB), white blood cells (WBCs), platelets and C-RP concentrations in the nosocomial infections, neonate sepsis and control group are presented in Tables 2(a)-(c). The nosocomial infection group showed normal blood parameters on the 1st day of medical treatment (on admission). On the 2nd day, $\mathrm{HB}$ and WBCs started to increase indicating microbial inflammation whereas platelets started to decrease. On the 3rd day, C-RP showed the highest measured value, 24 , indicating extreme infection. Then, the medical treatment continued day by day until the C-RP value reached the lowest value suggesting the patient was free of the infection. Nonetheless, neonatal sepsis (Table 2(b)) shows high values of WBCs and C-RP and the lowest PLT value on the 1st day of hospital admission indicating early sepsis.

\subsection{Antibiotic Microbial Response Tests}

Blood culture detected three different microbes: Staphylococcus aureus, E. coli, and Klebsiella spp. The percentage of Staphylococcus aureus infection was zero and $31.7 \%$ in nosocomial infections and neonate sepsis cases, respectively. The percentage of E. coli and Klebsiella spp. infections was $14.63 \%$ and $19.5 \%$ in nosocomial infections, respectively, whereas none were found in neonatal sepsis cases.

\subsection{Microbe Antibiotic Sensitivity Tests}

Microbe antibiotic sensitivity tests showed that E. coli is sensitive to Amikacin, Ciprofloxacin and Gentamicin; Klebsiella is sensitive to Amikacin, Meropenem and Vancomycin; and Staphylococcus aureus is sensitive to Amikacin, Ciprofloxacin, Meropenem and Cefotaxime.

\subsection{Management and Medical Follow-Up of the Cases}

The medical treatment continued as mentioned above until the C-RP values were reduced to the lowest value using the antibiotics mentioned above. It is obvious by looking at Table 2(b) that the value of platelets is low at high C-RP values, and the value tend to increase at a low $\mathrm{C}-\mathrm{RP}$ value. Comparing the data 
in Table 2(a) and Table 2(b) with those in Table 2(c), showing significant differences. This indicates that the blood parameters in Table 2(a) and Table 2(b) are higher than those in the control group (2C) indicating immediate medical treatments are required. Moreover, comparing blood parameters in Table 2(a) and Table 2(b) with those in Table 2(c) on the last day of medical treatment showing nearly similar parameters indicated effective follow-up and medical treatments. Statistical analysis showed significant differences between blood parameters in Table 2(a), 1st day and those in Table 2(c). Then, medical treatments continued until the statistical analysis showed a significant difference between the data in Table 2(a), especially at the 5th day and the 8th day of Table 2(b), and at the 5th day of Table 2(c), indicated successful medical treatments. So far, a regression examining the values of C-RP and platelets reveals a strong negative association with the $\mathrm{R} 2$ values of nosocomial infections and neonate sepsis cases, which were in the range $0.846-0.889$ (Figure 1).

Table 2. Blood parameters of nosocomial (2A), early sepsis (2B) and control group (2C).

(a)

\begin{tabular}{cccccc}
\hline \multirow{2}{*}{ Parameter } & \multicolumn{5}{c}{ Nosocomial } \\
\cline { 2 - 5 } & 1 1st d & 2nd d & 3rd d & 4th d & 5 th d \\
\hline HB $(\mathrm{g} / \mathrm{dl})$ & $18.30 \pm 0.57$ & $18.65 \pm 0.78$ & $16.70 \pm 0.28$ & $14.85 \pm 1.06$ & $15.35 \pm 0.78$ \\
WBCs $(\mathrm{k} / \mu \mathrm{l})$ & $13.40 \pm 0.85$ & $22.45 \pm 17.75$ & $17.30 \pm 14.14$ & $12.10 \pm 8.34$ & $12.95 \pm 10.54$ \\
Platelets $(\mathrm{k} / \mu \mathrm{l})$ & $219.50 \pm 5.59$ & $85.00 \pm 8.49$ & $80.50 \pm 3.61$ & $102.00 \pm 6.67$ & $182.50 \pm 4.75$ \\
C-RP $(\mathrm{mg} / \mathrm{dl})$ & $<6.00 \pm 0.00$ & $24.00 \pm 0.00$ & $18.00 \pm 8.49$ & $13.50 \pm 6.36$ & $5.15 \pm 0.92$ \\
\hline
\end{tabular}

(b)

\begin{tabular}{|c|c|c|c|c|c|c|c|c|}
\hline \multirow{2}{*}{ Parameter } & \multicolumn{8}{|c|}{ Neonatal sepsis } \\
\hline & 1 st d & 2nd d & $3 \mathrm{rd} \mathrm{d}$ & 4 th d & 5th d & 6th d & 7 th $d$ & 8th d \\
\hline $\mathrm{HB}(\mathrm{g} / \mathrm{dl})$ & $19.85 \pm 4.31$ & $17.05 \pm 1.34$ & $18.80 \pm 0.28$ & $19.25 \pm 1.77$ & $18.80 \pm 0.42$ & $19.25 \pm 1.77$ & $20.00 \pm 1.41$ & $19.30 \pm 1.84$ \\
\hline WBCs $(\mathrm{k} / \mu \mathrm{l})$ & $37.15 \pm 4.88$ & $28.55 \pm 9.12$ & $27.85 \pm 10.11$ & $21.80 \pm 1.27$ & $18.45 \pm 2.33$ & $30.20 \pm 6.79$ & $23.70 \pm 15.98$ & $16.45 \pm 6.29$ \\
\hline Platelets $(\mathrm{k} / \mu \mathrm{l})$ & $94.50 \pm 17.28$ & $105.50 \pm 14.18$ & $101.50 \pm 15.59$ & $103.00 \pm 12.02$ & $181.00 \pm 12.87$ & $91.00 \pm 13.44$ & $151.50 \pm 3.44$ & $261.00 \pm 2.73$ \\
\hline C-RP (mg/dl) & $24.00 \pm 0.00$ & $21.00 \pm 4.24$ & $18.00 \pm 8.49$ & $8.80 \pm 4.53$ & $6.75 \pm 7.42$ & $18.00 \pm 8.49$ & $5.80 \pm 0.28$ & $2.25 \pm 1.06$ \\
\hline
\end{tabular}

(c)

\begin{tabular}{cccccc}
\hline & \multicolumn{5}{c}{ Control group } \\
\cline { 2 - 6 } Parameter & 1 st d & 2nd d & 3rd d & 4th d & 5 th d \\
\hline HB $(\mathrm{mg} / \mathrm{dl})$ & $16.12 \pm 2.45$ & $15.81 \pm 1.51$ & $16.73 \pm 2.45$ & $18.51 \pm 0.45$ & $16.63 \pm 0.86$ \\
WBC $(\mathrm{k} / \mu \mathrm{l})$ & $18.23 \pm 1.23$ & $20.22 \pm 3.34$ & $23.12 \pm 5.63$ & $23.41 \pm 6.42$ & $21.00 \pm 2.12$ \\
Platelets $(\mathrm{k} / \mu \mathrm{l})$ & $210.55 \pm 21.64$ & $250.60 \pm 14.32$ & $220.78 \pm 8.94$ & $197.98 \pm 5.43$ & $217.50 \pm 21.65$ \\
C-RP $(\mathrm{mg} / \mathrm{dl})$ & $3.24 \pm 0.11$ & $2.80 \pm 0.23$ & $3.22 \pm 0.87$ & $2.11 \pm 0.67$ & $3.30 \pm 0.44$ \\
\hline
\end{tabular}




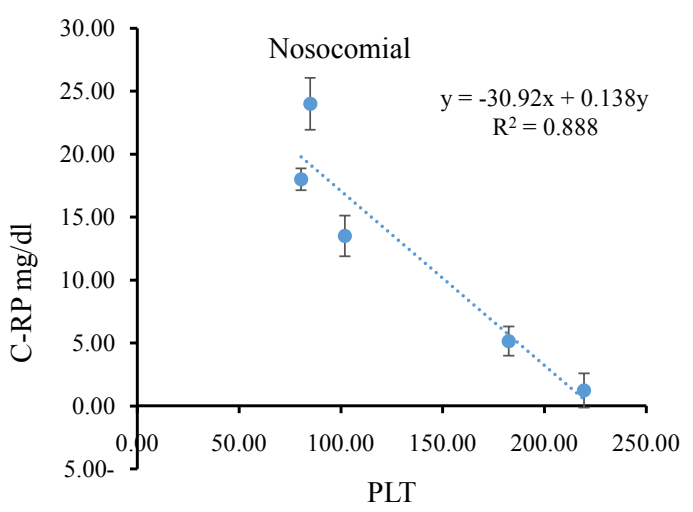

Early sepsis

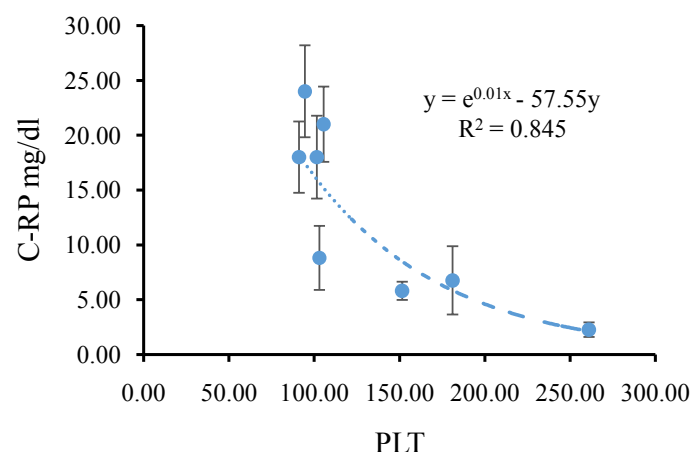

Figure 1. Dynamic relationship between C-RP and platelets concentrations in blood serum of sepsis cases.

\section{Discussions}

The data in Table 1 clearly show the physical and clinical features of nosocomial infections, neonatal sepsis cases and the control group on the 1st day of medical treatments. It is obvious that the body weight on patients with nosocomial infections ranged from $1.75-2.8 \mathrm{~kg}$. The lowest body weight $(1.75 \mathrm{~kg})$ may be caused by the fact that the neonate was born at 32 weeks, several weeks before a normal gestational period (38 weeks). The body temperature of the groups ranged from $36^{\circ} \mathrm{C}-39^{\circ} \mathrm{C}$, suggesting the existence of hypothermia and hyperthermia among the groups. The skin color varied from pink to cyanotic. The activity was low in nosocomial infection cases and hypoactive in sepsis. Feeding was performed by nasogastric tube in all septic cases, whereas breastfeeding occurred within the control group. Furthermore, the chest suffered from lung collapse in nosocomial infections, whereas no wheezing was observed in cases of neonate sepsis or the control group. Additionally, cardiomegaly was observed in three cases, including one case in patients with nosocomial infections and two in patients with neonate sepsis. No cases were observed within the control group. Moreover, investigation of the abdomen showed a similar percentage of hepatomegaly in nosocomial infections and neonate sepsis. The heartbeats were in the range of $48-146$ beats per minute. The quite low heartbeats may be due to the fact the sympathetic and parasympathetic nervous system are affected by the bacterial toxins in which 
these two systems have tremendous effect on controlling the heart rate. It is well known in the literature that neonatal and nosocomial sepsis can decrease heart beats.

The percentage of jaundice in nosocomial infections was the lowest, whereas the highest percentage was found within the control group. In all cases, the central nervous system does not show any abnormal movement.

The explanation of these results is that patients with nosocomial infections and neonate sepsis were infected with E. coli, Klebsiella or Staphylococcus aureus (Table 1). These microbes produced toxins in the blood that disturbed the normal life of the baby. Moreover, the neonates were given antibiotics without performing a blood culture or C-RP measurement. It appears that Staphylococcus aureus, E. coli, and/or Klebsiella caused sepsis and septic shocks to many cases due to the toxins they produced on the hosting body. Our explanation agrees with Butt et al. [21] who found that Staphylococcus aureus and E. coli were a common causative agent of postoperative infections and puerperal sepsis. Nevertheless, gram negative bacilli were found in larger numbers in nosocomial indication than in neonatal sepsis probability due to the fact that $E$. coli can be found in contaminated drinking water and or food samples.

Furthermore, the mistaken medical treatment may result in antibiotic-resistant Staphylococcus aureus, which causes a major bloodstream infection with a high mortality rate among neonates. Additionally, a continuous high level of C-RP would have occurred (Table 2). Thus, C-RP values remain at high levels as long as the neonate receives ineffective antibiotics. This finding is in accordance with Jamsa et al. [22] who found high values of C-RP during sepsis. By performing blood cultures and microbial sensitivity tests, the neonates would have received effective antibiotics that reduced or stopped the growth of microbes. This would have caused the values of C-RP to be progressively reduced and platelets to increase. The right medical treatment helps the neonates and makes them active again where they are able to breastfeed instead of having a nasogastric tube for feeding. It is well known that neonatal sepsis remains an important cause of morbidity and mortality and requires prompt empiric treatment.

It can be suggested that neonatal septic cases should undergo blood culture for detecting the corresponding microbes, microbial-antibiotics sensitivity test and $\mathrm{C}-\mathrm{RP}$ measurements during hospital admission time and the follow-up medical period. If the C-RP value is reduced during the follow-up treatment, the medical treatment goes in the right direction, and the neonate will successfully be saved. On the other hand, if the C-RP value remains higher than $6 \mathrm{mg} / \mathrm{dl}$ during the medical treatment, steps such as changing antibiotics and checking for multi-resistant microbes should be undertaken. During medical treatment, the blood parameters of the patients should be compared with the blood parameters in the control group (Table 2(c)) using statistical analysis to detect differences. If the $\mathrm{p}$-value is above 0.05 , this indicates that there are no significant differences between the control group and patients. If the p-value is equal or less than 0.05 , this indicates that some blood parameter of the patient is still higher than in the 
control group, and medical treatment is still required. Furthermore, the data in Figure 1 show a strong negative association between C-RP and platelet values in the blood. Moreover, this figure supports the abovementioned discussion. So far, our discussion is in agreement with Kim et al. [23], who investigated the clinical and microbiological aspects associated with early patient mortality from methicillin-resistant Staphylococcus aureus bacteremia. Further support to our discussion comes from previous reports [24] [25] which investigated early recognition of methicillin-resistant Staphylococcus aureus surgical site infections, and revealed that there was positive consensus that sepsis, $>2$ weeks of hospitalization, was one of the major factors.

However, the presented results clearly demonstrate the effectiveness of blood culture, microbe-antibiotic sensitivity tests, and C-RP measurements as critical parameters for optimal and successful medical treatments.

\section{Conclusion}

Neonatal and nosocomial sepsis are extremely difficult cases. They need careful attention and a blood culture investigation to identify the microbes and its respective active antibiotics before any antibiotic is used in the septic cases. C-RP kinetic measurements show the effectiveness of medical treatments. It is strongly recommended to make a kinetic C-RP measurement during the follow-up treatment. In summary, blood culture identification, bacterial antibiotic activity and C-RP are important driving parameters in septic case treatments.

\section{Declarations}

\section{Ethics Approval and Consent to Participate}

Ethical approval of this study was obtained from the Research Committee of the Faculty of Science and Faculty of Medicine, The Islamic University Gaza.

\section{Consent Form}

Consent forms were obtained from the father and mother of neonates.

\section{Availability of Data and Material}

The datasets supporting the conclusions of this article are included within the article, can be available online after publication or under request to the authors.

\section{Author Contribution Statement}

YZE designed the experiments and wrote the manuscript; ATA performed the experiments; MRA performed the statistical analysis. All authors reviewed the manuscript.

\section{Acknowledgements}

Special thanks to the Alexander von Humboldt Foundation for a Research Fellowship at Leipzig University and the BAM Institute Germany. 


\section{Authors' Information}

YZE (Ph.D.) is a Professor of toxicology, Faculty of Science, The Islamic University-Gaza. ATA (MSc), and MRA (Ph.D) is a Professor of Geochemistry, Faculty of Science, The Islamic University Gaza.

\section{Conflicts of Interest}

The authors declare no conflicts of interest regarding the publication of this paper.

\section{References}

[1] Varpula, M., Karlsson, S., Parviainen, I., et al. (2007) Community-Acquired Septic Shock: Early Management and Outcome in a Nationwide Study in Finland. Acta Anaesthesiologica Scandinavica, 51, 1320-1326. https://doi.org/10.1111/j.1399-6576.2007.01439.x

[2] Kumar, A., Roberts, D., Wood, K.E., et al. (2006) Duration of Hypotension before Initiation of Effective Antimicrobial Therapy Is the Critical Determinant of Survival in Human Septic Shock. Critical Care Medicine, 34, 1589-1596. https://doi.org/10.1097/01.CCM.0000217961.75225.E9

[3] Dellinger, R.P., Levy, M.M., Rhodes, A., et al. (2013) Surviving Sepsis Campaign: International Guidelines for Management of Severe Sepsis and Septic Shock. Critical Care Medicine, 41, 580-587.

[4] Schecter, A., Papke, O., Ryan, J., Furst, P., Isaac, J., Hrimat, N., Neiroukh, F., Safi, J., El-Nahhal, Y., Abu El-Haj, S., Avni, A., Richter, E., Chuwers, P. and Fischbein, A. (1997) Dioxins, Dibenzofurans and PCBs in Human Blood, Human Milk and Food from Israel, the West Bank and Gaza. Organohalogen Compounds, 33, 457-461.

[5] El-Nahhal, Y. (2017) Risk Factors among Greenhouse Farmers in Gaza Strip. Occupational Diseases and Environmental Medicine, 5, 1-10. https://doi.org/10.4236/odem.2017.51001

[6] Marnell, L., Mold, C. and Du Clos, T.W. (2005) C-Reactive Protein: Ligands, Receptors and Role in Inflammation. Clinical Immunology, 117, 104-111. https://doi.org/10.1016/j.clim.2005.08.004

[7] Agassandian, M., Shurin, G.V., Ma, Y. and Shurin, M.R. (2014) C-Reactive Protein and Lung Diseases. The International Journal of Biochemistry \& Cell Biology, 53, 77-88. https://doi.org/10.1016/j.biocel.2014.05.016

[8] Shaaban, R., Kony, S., Driss, F., Leynaert, B., Soussan, D., Pin, I., et al. (2006) Change in C-Reactive Protein Levels and FEV1 Decline: A Longitudinal Population-Based Study. Respiratory Medicine, 100, 2112-2120. https://doi.org/10.1016/j.rmed.2006.03.027

[9] Trulock, E.P., Christie, J.D., Edwards, L.B., Boucek, M.M., Aurora, P., Taylor, D.O., et al. (2007) Registry of the International Society for Heart and Lung Transplantation: Twenty-Fourth Official Adult Lung and Heart-Lung Transplantation Report 2007. The Journal of Heart and Lung Transplantation, 26, 782-795. https://doi.org/10.1016/j.healun.2007.06.003

[10] Kikuchi, K., Kohyama, T., Yamauchi, Y., Kato, J., Takami, K., Okazaki, H., et al. (2009) C-Reactive Protein Modulates Human Lung Fibroblast Migration. Experimental Lung Research, 35, 48-58. https://doi.org/10.1080/01902140802404138

[11] Agusti, C., Rano, A., Rovira, M., Filella, X., Benito, N., Moreno, A., et al. (2004) In- 
flammatory Response Associated with Pulmonary Complications in Non-HIV Immunocompromised Patients. Thorax, 59, 1081-1088. https://doi.org/10.1136/thx.2004.030551

[12] Kony, S., Zureik, M., Driss, F., Neukirch, C., Leynaert, B. and Neukirch, F. (2004) Association of Bronchial Hyperresponsiveness and Lung Function with C-Reactive Protein (C-RP): A Population Based Study. Thorax, 59, 892-896. https://doi.org/10.1136/thx.2003.015768

[13] Calero, C., Arellano, E., Lopez-Villalobos, J.L., Sánchez-López, V., Moreno-Mata, N. and López-Campos, J.L. (2014) Differential Expression of C-Reactive Protein and Serum Amyloid A in Different Cell Types in the Lung Tissue of Chronic Obstructive Pulmonary Disease Patients. BMC Pulmonary Medicine, 14, 95. https://doi.org/10.1186/1471-2466-14-95

[14] Zhou, B., Liu, J., Wang, Z.M. and Xi, T. (2012) C-Reactive Protein, Interleukin 6 and Lung Cancer Risk: A Meta-Analysis. PLOS ONE, 7, e43075. https://doi.org/10.1371/journal.pone.0043075

[15] Razi, E.E.H., Akbari, H., Chavoshi, V. and Razi, A. (2012) Evaluation of High-Sensitivity C-Reactive Protein in Acute Asthma. Tanaffos, 11, 32-37.

[16] Ellahony, D.M., El-Mekkawy, M.S. and Farag, M.M. (2017) A Study of Red Cell Distribution Width in Neonatal Sepsis. Pediatric Emergency Care. https://doi.org/10.1097/PEC.0000000000001319

[17] Kakinoki, H., Tobu, S., Kakinoki, Y., Udo, K., Uozumi, J. and Noguchi, M. (2017) Risk Factors for Uroseptic Shock in Patients with Urolithiasis-Related Acute Pyelonephritis. Urologia Internationalis, 100, 37-42. https://doi.org/10.1159/000481801

[18] WHO (2013) Evaluation for Severe Sepsis Screening Tool. Version 7.2.

[19] Palestinian National Authority Ministry of Health (2004) Postpartum Care Protocols.

[20] Bone, R.C., Grodzin, C.J. and Balk, R.A. (1997) Sepsis: A New Hypothesis for Pathogenesis of the Disease Process. Chest, 112, 235-243.

https://doi.org/10.1378/chest.112.1.235

[21] Butt, I.J., Khan, S., Butt, S. and Bhutta, S. (2013) Frequency and Treatment of Methicillin Resistant Staphylococcus aureus in Obstetric and Gynaecological Sepsis. Journal of College of Physicians and Surgeons Pakistan, 23, 708-710.

[22] Jämsä, J., Ala-Kokko, T., Huotari, V., et al. (2017) Neutrophil CD64, C-Reactive Protein, and Procalcitonin in the Identification of Sepsis in the ICU-Post-Test Probabilities. Journal of Critical Care, 43, 139-142. https://doi.org/10.1016/j.jcrc.2017.08.038

[23] Kim, T., Chong, Y.P., Park, K.H., Bang, K.M., Park, S.J., Kim, S.H., Jeong, J.Y., Lee, S.O., Choi, S.H., Woo, J.H. and Kim, Y.S. (2017) Clinical and Microbiological Factors Associated with Early Patient Mortality from Methicillin-Resistant Staphylococcus aureus Bacteremia. The Korean Journal of Internal Medicine, 34, 184-194. https://doi.org/10.3904/kjim.2016.351

[24] Sganga, G., Tascini, C., Sozio, E. and Colizza, S. (2017) Early Recognition of Methicillin-Resistant Staphylococcus aureus Surgical Site Infections Using Risk and Protective Factors Identified by a Group of Italian Surgeons through Delphi Method. World Journal of Emergency Surgery, 12, 25. https://doi.org/10.1186/s13017-017-0136-3

[25] El-Nahhal, Y. and Al shareef, A. (2018) Effective Biomarkers for Successful Management of Sepsis. Trends in Medicine, 18, 1-8.

https://doi.org/10.15761/TiM.1000156 


\section{Abbreviations}

HB: Hemoglobin;

WBC: White Blood Cells;

CBC: Complete Blood Count;

PLT: Platelets;

C-RP: C-Reactive Protein;

WHO: World Health Organization. 